\title{
Spontaneous Pneumothorax due to Ectopic Deciduosis: A Case Report
}

\author{
Wojciech Dudek ${ }^{1}$ Waldemar Schreiner ${ }^{1}$ Johanna Strehl ${ }^{2}$ Horia Sirbu ${ }^{1}$ \\ ${ }^{1}$ Department of Thoracic Surgery, University Hospital of Erlangen, \\ Erlangen, Germany \\ 2 Department of Pathology, University Hospital of Erlangen, Erlangen, \\ Address for correspondence Wojciech Dudek, MD, Department of \\ Thoracic Surgery, University Hospital of Erlangen, Krankenhausstr. 12, \\ Erlangen 91054, Germany (e-mail: wojciech.dudek@doctors.org.uk).
} Germany

Thorac Cardiovasc Surg Rep 2014;3:58-60.

\begin{abstract}
Keywords

- pleuropulmonary ectopic deciduosis

- spontaneous pneumothorax

- thoracoscopic surgery

This report presents a 20-week pregnant 38-year-old woman with right-sided pneumothorax due to pulmonary deciduosis. Initial pleural drainage was ineffective. Videoassisted thoracoscopy revealed areas of consolidation within the lung parenchyma. A wedge resection with partial pleurectomy was performed. Histopathological examination showed subpleural decidual implants. The patient made a full recovery and was discharged on day 5 . Videoscopic inspection of the lung parenchyma and pleura with resection of decidual foci is the recommended treatment for pneumothorax in pregnant women with pleuropulmonary deciduosis in whom classical pleural drainage is ineffective.
\end{abstract}

\section{Case Report}

Ectopic deciduosis is a common finding in pregnancy but its pleuropulmonary manifestation is extremely rare. Its equivalent in nonpregnant women of childbearing age is pleuropulmonary endometriosis which may result in catamenial pneumothorax.

A 20-week pregnant 38-year-old primigravida presented to the medical emergency department approximately 3 weeks after the onset of mild to moderate shortness of breath. She had a history of endometriosis, hypothyreosis, and suspicion of von Willebrand syndrome. Pregnancy was achieved with the help of in vitro fertilization.

On admission, the patient's observations were within normal range. Chest examination revealed a central trachea with hyperresonant percussion and reduced air entry on the right side. Heart sounds were clear. A subsequent chest radiogram demonstrated a right-sided pneumothorax (-Fig. 1). A 28 Ch chest drain was placed and the patient was admitted to the medical ward. After 19 days of unsuccessful drainage with a $20 \mathrm{~cm} \mathrm{H_{2 }}$ O suction, the right lung remained partially collapsed. Subsequently, the patient was referred to the thoracic surgical team and listed for a video- assisted thoracoscopic procedure the following day. Four well-demarcated, bluish consolidations of the lung parenchyma with ruptured multiple small blebs (two within the right upper lobe and two in the middle lobe) were identified intraoperatively and wedge-excised using an endostapler. The right hemidiaphragm appeared normal. An apicalodorsal pleurectomy was performed and a single chest drain was inserted. The postoperative course was uneventful and the patient was discharged from the surgical unit on the 5th postoperative day. Pathological examination of the resected lung parenchyma showed decidual implants in the periphery of subpleural located hollow spaces of up to $0.3 \mathrm{~cm}$ (-Fig. 2). The adjacent tissue was fibrotic, with a high content of elastic fibers. In the visceral as well as parietal pleura, chronic inflammation and a pronounced infiltrate of eosinophils were seen. The further course of the pregnancy was uneventful with no recurrence of pneumothorax. The baby was delivered via cesarean section.

\section{Discussion}

We report on a very rare case of spontaneous pneumothorax due to ectopic deciduosis in a pregnant woman. A common received

December 30, 2013

accepted after revision

April 11, 2014

published online

September 16, 2014
DOI http://dx.doi.org/

10.1055/s-0034-1383511. ISSN 2194-7635.
License terms

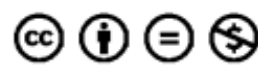

(c) 2014 Georg Thieme Verlag KG
Stuttgart . New York 


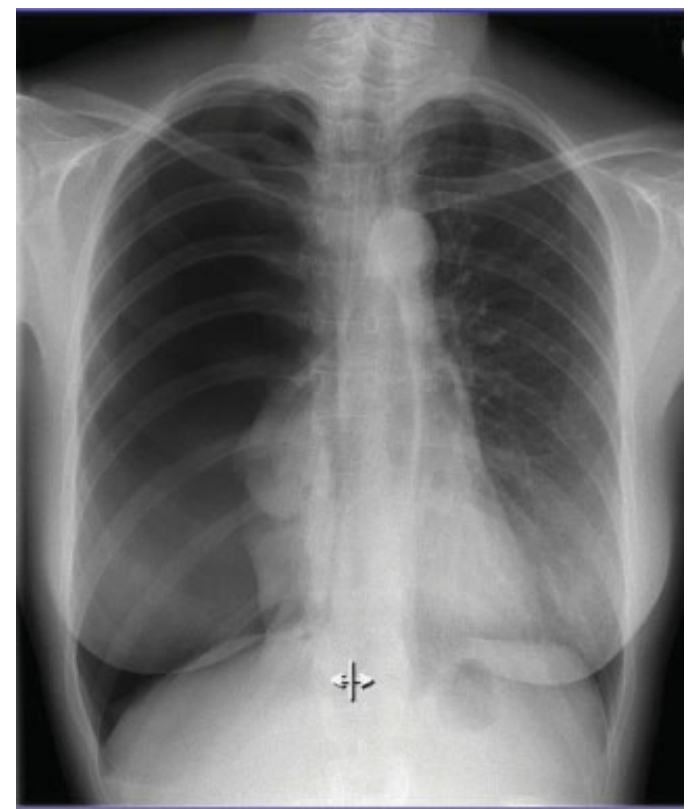

Fig. 1 Chest roentgenogram of our patient shows a massive rightsided pneumothorax.

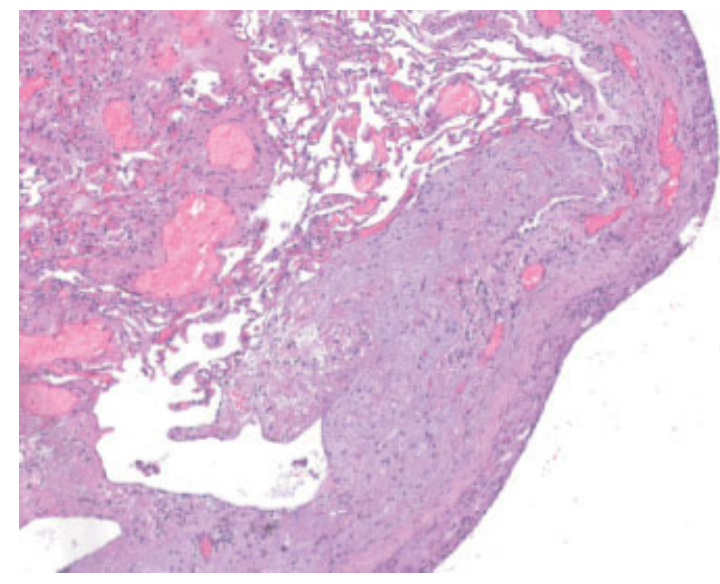

Fig. 2 Decidual implants in the lung parenchyma.

cause of spontaneous pneumothorax in women is the so-called catamenial (from Greek "monthly") pneumothorax-its incidence may be as high as $35 \%$ of all pneumothorax cases in women of childbearing age. ${ }^{1}$ In our patient, the pneumothorax occurred during physiological amenorrhea and therefore, cannot be called "catamenial" according to the definition.

To our knowledge, only three histological proven cases of pneumothorax due to pleural/pulmonary deciduosis and endometriosis during pregnancy have been reported to date in the English language literature. Kim et al described a case of a 34-year-old 21-week pregnant primigravida successfully treated for recurrent pneumothorax with video-assisted resection of the decidual implants from the middle lobe and right hemidiaphragm, with closure of the diaphragmatic fenestration using cellulose mesh. They stated that the coexisting diaphragmatic and pulmonary ectopic deciduosis probably resulted from pregnancy-induced decidual changes of the ectopic endometrium. ${ }^{2}$ Yoshioka et al reported a recurrence of spontaneous pneumothorax due to pleural endometriosis in a 29-year-old 8-week pregnant woman. After ineffective pleural drainage she was treated operatively via video-assisted excision of the multiple endometrial implants from the visceral pleura. ${ }^{3}$ Flieder et al described pulmonary ectopic deciduosis as a result of the pregnancy-induced transformation of the longstanding pulmonary stromal endometriosis in a pregnant woman with spontaneous pneumothorax. ${ }^{4}$

Pulmonary deciduosis probably develops through the decidualization of preexisting endometrial implants in the lung parenchyma during pregnancy. The mechanism by which decidualization of endometriotic lesions occurs is not yet known. In the case of our patient, there was no diagnosis of pulmonary endometriosis before the operative therapy of the pneumothorax. However, 4 years before the pneumothorax occurred, the patient underwent a resection of the sigma and upper rectum due to deeply infiltrating endometriosis. The pathological work-up showed extensive endometriosis penetrating deeply into the muscularis propria of the intestinal wall. It may be speculated that the propensity of this severe form of endometriosis to infiltrate deeply into the tissue may have played a role in the extraabdominal spread of endometriosis implants. Furthermore, our patient underwent in vitro fertilization to conceive. Ovarian stimulation with subsequent high levels of estrogen may also have contributed to the development of the ectopic endometriosis implants.

Current guidelines for management of the endometriosis recommend inhibition of the endometrial activity and therefore, all hormonal therapeutic strategies (GnRH agonists, Dienogest) target to induce amenorrhea, suppress ovulation and subsequently inhibit the cyclical changes of the endometrium. Pregnancy as a physiological amenorrhea is also a decelerator of endometriosis. Even so, as demonstrated in the present case, endometriosis-related problems may arise in pregnancy. The reasons for this are unclear. Setúbal et al published a small series of three pregnant women who suffered a bowel perforation caused by deep endometriosis during pregnancy or in the immediate postpartum period. In addition, they identified 12 comparable cases in the English literature. The authors speculate that the decidualization of endometriosis during pregnancy may lead to a structural weakening of the host tissue and thus give rise to bowel perforations. ${ }^{5} \mathrm{~A}$ similar mechanism may be responsible for endometriosis-related pneumothorax in pregnancy. However, as of now, there is no conclusive data regarding the biology of deep infiltrating endometriosis during pregnancy. Research into this matter is hampered by the rather small number of cases and the difficulty of clinically recognizing endometriosis-related problems in pregnancy.

Pneumothorax associated with pleural/pulmonary implants of the decidua in the pregnant patient can be managed in a similar manner to other types of pneumothorax during 
pregnancy. Provided the mother is not short of breath, there is no fetal distress and the pneumothorax is less than $2 \mathrm{~cm}$, simple observation is recommended. ${ }^{6}$ Otherwise, aspiration can be performed. Current guidelines advise prolonged chest drainage only by persistent air leak; this should be maintained to near term. Because of the risk of recurrence in subsequent pregnancies, an elective surgical procedure should be performed after convalescence. In our patient who remained dyspneic despite active chest drainage and showed a persistent air leak with approximately 15 weeks remaining to delivery, the decision was made to perform a video-assisted procedure. Video-assisted thoracic surgery (VATS) exploration is a standard first-line surgical management of the endometriosis-related pneumothorax. During the VATS procedure, the surface of the lung, parietal pleura, and hemidiaphragm should be thoroughly inspected and all suspicious lesions wedge excised. ${ }^{7}$ Pleurectomy, including diaphragmatic pleura, should also be performed. In pregnant women, talcum pleurodesis is strongly contraindicated due to the risk of embolism. For diaphragmatic localization of the endometriotic implants, plication and/or reinforcement with mesh is recommended.

\section{References}

1 Legras A, Mansuet-Lupo A, Rousset-Jablonski C, et al. Pneumothorax in women of child-bearing age: an update classification based on clinical and pathologic findings. Chest 2014;145(2): 354-360

2 Kim YD, Min KO, Moon SW. Thoracoscopic treatment of recurrent pneumothorax in a pregnant woman: a case of ectopic deciduosis. Thorac Cardiovasc Surg 2010;58(7):429-430

3 Yoshioka H, Fukui T, Mori S, Usami N, Nagasaka T, Yokoi K. Catamenial pneumothorax in a pregnant patient. Jpn J Thorac Cardiovasc Surg 2005;53(5):280-282

4 Flieder DB, Moran CA, Travis WD, Koss MN, Mark EJ. Pleuropulmonary endometriosis and pulmonary ectopic deciduosis: a clinicopathologic and immunohistochemical study of 10 cases with emphasis on diagnostic pitfalls. Hum Pathol 1998;29(12): 1495-1503

5 Setúbal A, Sidiropoulou Z, Torgal M, Casal E, Lourenço C, Koninckx P. Bowel complications of deep endometriosis during pregnancy or in vitro fertilization. Fertil Steril 2014;101(2):442-446

6 MacDuff A, Arnold A, Harvey J; BTS Pleural Disease Guideline Group. Management of spontaneous pneumothorax: British Thoracic Society Pleural Disease Guideline 2010. Thorax 2010;65 (Suppl (Suppl 2):ii18-ii31

7 Marshall MB, Ahmed Z, Kucharczuk JC, Kaiser LR, Shrager JB. Catamenial pneumothorax: optimal hormonal and surgical management. Eur J Cardiothorac Surg 2005;27(4):662-666 